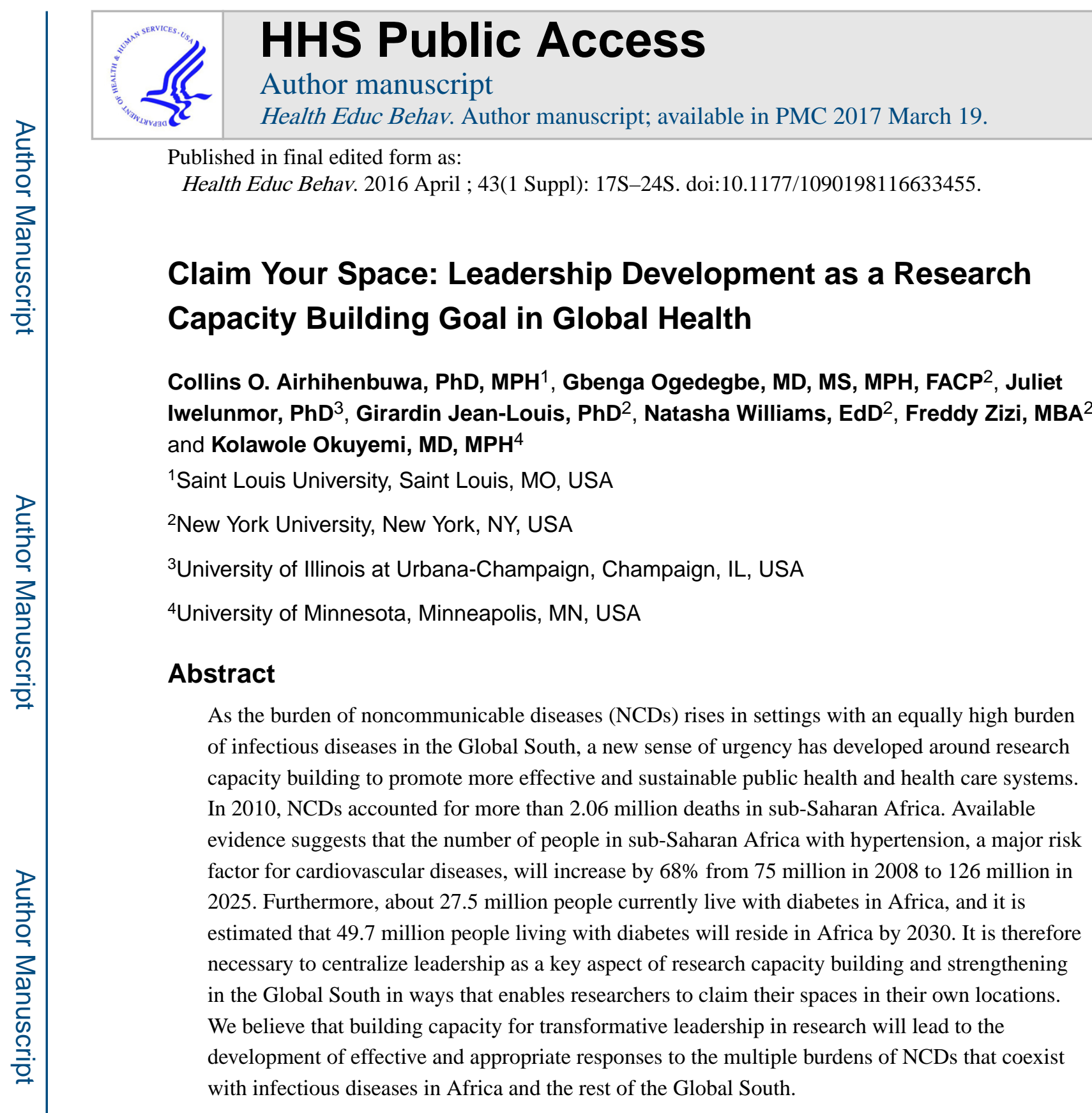

\title{
Keywords
}

Africa; capacity building; Global South; leadership; NCDs

Reprints and permissions: sagepub.com/journalsPermissions.nav

Corresponding Author: Collins O. Airhihenbuwa, Saint Louis University, 3545 Lafayette Avenue, Saint Louis, MO 63104, USA. airhihenbuwaco@slu.edu.

Declaration of Conflicting Interests

The authors declared no potential conflicts of interest with respect to the research, authorship, and/or publication of this article. Supplement Issue Note

This article is part of a Health Education \& Behavior supplement, "Noncommunicable Diseases in Africa and the Global South," which was supported by SAGE Publishing, with additional support from the National Heart, Lung, and Blood Institute Contract No. HHSN268201500073P. The entire supplemental issue is available open access for one year at http://heb.sagepub.com/content/ 43/1_suppl.toc. 
As the burden of noncommunicable diseases (NCDs) rises in settings with an equally high burden of infectious diseases (IDs) in the Global South, ${ }^{1}$ a new sense of urgency has developed around research capacity building in order to promote more effective and sustainable public health and health care systems (C. O. Airhihenbuwa et al., 2011; Drain et al., 2007; Lansang \& Dennis, 2004; Raeburn, Akerman, Chuengsatiansup, Mejia, \& Oladepo, 2006). The statistics are startling. In 2010, NCDs accounted for more than 2.06 million deaths in sub-Saharan Africa (Naghavi \& Forouzanfar, 2013). Available evidence suggests that the number of people in sub-Saharan Africa with hypertension, a major risk factor for cardiovascular diseases, will increase by 68\% from 75 million in 2008 to 126 million in 2025 (Mayosi, 2013). Furthermore, about 27.5 million people currently live with diabetes in Africa, and it is estimated that 49.7 million people living with diabetes will reside in Africa by 2030 (Mayosi, 2013). Obesity, a well-known risk factor for many chronic diseases, is also on the rise in sub-Saharan Africa, with as much as $20 \%$ to $50 \%$ of urban populations in Africa currently classified as overweight or obese (Ziraba, Fotso, \& Ochako, 2009). David Hunter and Srinath Reddy articulated the sense of urgency around addressing this rising NCD epidemic in the Global South (Hunter \& Reddy, 2013). More recently, a special issue of the journal $A I D S$ outlined the need to address the "double burden" of IDs and NCDs in the Global South, given the multiple burdens of diabetes and hypertension among the same population that carries the heaviest burdens of tuberculosis and HIV and AIDS (Geneau \& Hallen, 2012).

While there is an urgent need to reprioritize NCDs to be as important as IDs in the Global South, a similar urgency is required to prioritize capacity building to address NCDs, even in areas where the current health system is designed to prioritize either IDs or NCDs (Narayan et al., 2014). An important and even more crucial requirement for addressing capacity building is the mismatch between this increased burden and the research capacities of countries in the Global South to lead in generating new knowledge to combat this dual burden of diseases (Nchinda, 2002). It is therefore necessary to prioritize leadership as central to research capacity building and strengthening in the Global South, since knowledge production enables researchers to claim spaces in their own locations. We believe that building capacity for transformative leadership in research will lead to the development of effective and sustainable responses to the multiple burdens of NCDs in Africa and the rest of the Global South.

\section{Why Leadership?}

He who is leading and has no one following is only taking a walk. (African proverb)

Definitions of leadership abound. When we refer to leadership in this article, we refer to the capacity to advance values and skills that are critical for researchers in the Global South to advance new and innovative solutions to the persistent inequity that continues to increase disease burdens for the local population. To achieve the goals of leadership, the number of

\footnotetext{
${ }^{1}$ These are countries with low per capita income $(<\$ 9,000$ per year) and low Human Development Indices $(<0.8$ HDI $)$; most of these countries are located in the Southern Hemisphere.
} 
scholars who participate actively in research production must first be increased. Although countries in the Global South report an increased burden of NCDs, the amount of research originating from these countries is low (Chu, Jayaraman, Kyamanywa, \& Ntakiyiruta, 2014; Langer, Díaz-Olavarrieta, Berdichevsky, \& Villar, 2004). For example, in 2014, sub-Saharan African researchers produced only $1.3 \%$ of biomedical research in high-impact peerreviewed journals (Uthman et al., 2015).

Advancements in the production and application of scientific knowledge have the following four prerequisites: (1) individual research skills, (2) appropriate infrastructure, (3) relevance to national policies, and (4) the ability to contribute to global research and policy (Chu et al., 2014; Trostle \& Simon, 1992). Meeting any (let alone all) of these prerequisites is profoundly challenging for researchers in countries in the Global South, particularly due to difficulties associated with the research and publication process, editorial bias, and information access (Nchinda, 2002; Trostle \& Simon, 1992). Institutions in many countries lack appropriate self-sustaining research capacity, in terms of both the number, quality, and cultural relevance of trained researchers and the appropriate institutional capacity for producing research that affects national health priorities (Nchinda, 2002; Trostle \& Simon, 1992). It is no surprise that there are a limited number of trained researchers with relevant skills in contexts with poor funding and infrastructure (e.g., designated local health and medical research funding agencies, computers and relevant software for data analysis), and that limited research is being produced that can influence policy (Nchinda, 2002). Moreover, there are problems with access to peer-reviewed literature with a consistent focus on local realities, resulting in limited expertise in preparing articles that have relevance to local populations. Given limited editorial representation of scholars with research commitments to their regions, many researchers in the Global South face difficulties in translating their findings into policies that will make a difference in their own countries (Trostle \& Simon, 1992; Uthman et al., 2015). Moreover, these same researchers have difficulties disseminating their findings to a global audience, which means that most of the studies authored by the few scholars in the Global South who publish actively are often not included in major databases. Yet the information, research capacity, and leadership needed to develop, translate, and disseminate this information are crucial to improving overall health and wellbeing in these regions.

Since the late 1990s, there have been calls to increase research capacity among health scholars in the Global South. At the 1998 World Health Assembly, Dr. Gro Bruntland, the then Director General of the World Health Organization, said in her address that "capacitybuilding in countries in greatest need must remain a priority issue for the international community" (Nchinda, 2002, p. 1701). Since then, efforts have been made to implement appropriate training to improve scientists' abilities to undertake quality research and provide institutional support, equipment, supplies, and other logistical support to the institutions that employ trained scientists. One major challenge is that funding for research capacity building has been very limited. Capacity building for research ranges from training graduate students (C. O. Airhihenbuwa et al., 2011) to training early career scholars during their formative years in research positions. In one research capacity building project related to HIV and AIDS stigma in South Africa led by the lead author (COA), 30 postgraduate students and 6 faculty/staff were trained over 5 years to employ the PEN-3 cultural model in the 
development of research to address HIV- and AIDS-related stigma (C. Airhihenbuwa et al., 2009). This research collaboration was between the Pennsylvania State University, the Human Sciences Research Council of South Africa, the University of the Western Cape of South Africa, and the University of Limpopo of South Africa. The success of the project was credited to a partnership that was based on mutual respect and openness to learning from each other (C. O. Airhihenbuwa et al., 2011).

Building research capacity by training early or midcareer health professionals creates possibilities for sustainability, as was the case with the Fogarty-funded Cardiovascular Research Training (CaRT) Institute in Ghana. CaRT, led by the co-lead author (GO), is a collaborative training program between the New York University School of Medicine, the Loyola University School of Medicine, and the University of Ghana School of Public Health. The CaRT Institute, which is located in Ghana, is geared toward developing junior faculty, postgraduate physicians, and matriculated $\mathrm{MPH} / \mathrm{PhD}$ graduate students as independent investigators. The long-term training model requires mentees to attend the program for a period of 2 years, with the first year spent attending intensive courses, including fundamentals of research methodology, health services research, comparative effectiveness research, and dissemination and implementation research. During the second year, mentees spend their time participating in various career development and mentorship activities and honing their acquired research skills as they develop and implement individual research projects and prepare manuscripts and grant applications. To date, 64 scholars have been trained in the CaRT program. Since the inception of the program in 2012, CaRT scholars have published dozens of articles in peer-reviewed journals and presented scientific papers at over 30 conferences. In addition, some of these trained early career scholars have received funding to support their work at levels that have already exceeded the expectations established for the training grant. While the long-term goal of CaRT is to produce a sustainable network of individuals who are well trained in various aspects of cardiovascular and NCD research, achieving this goal will require transformative leadership that will enable scholars to claim their own spaces and commit to reaffirming the spaces in which they and their protégés advance Global South research agendas. We believe that this leadership is central to research capacity building and strengthening if we are to achieve a meaningful population health impact in reducing the burden of NCDs and IDs globally.

Indeed, with the increasing realization that local contexts are situated within the global community, capacity building and leadership efforts in the Global South can be linked to similar efforts in the Global North. For example, considerable resources are being invested to increase the number of health and medical scientists from underrepresented populations in the United States, given the national priority to reduce health disparities. One such example is the PRIDE program funded by the National Health, Lung, and Blood Institute. The PRIDE Institute at New York University, led by two of the coauthors (GJ, GO), provides a unique opportunity to build and sustain a global academic workforce for capacity building, a fundamental component in programs designed to improve health equity among vulnerable populations (Jean-Louis et al., 2015). The PRIDE program offers mentored research training to underrepresented early career investigators, which is essential for strengthening and developing a competent and diverse academic global workforce (Guilbert, 2006), as well as increasing knowledge related to capacity building as well as exposure to global programs 
that utilize task shifting principles (Ogedegbe, Gyamfi, et al., 2014; Ogedegbe, PlangeRhule, et al., 2014); the program also identifies other key solutions, including grant funding. These efforts are particularly crucial for the academic workforce, as these skills and strategies are important when building collaborations with local organizations and establishing long-term and meaningful partnerships. Additionally, these programs promote leadership through mentoring and exposure to academic leaders engaged in capacity building and global health, thus increasing confidence by empowering academic faculty to allow their passions to drive their scholarly pursuits.

One of the key components of leadership in research capacity building that is shared by $\mathrm{CaRT}$ and PRIDE is the exposure of trainees to the role of culture in research. Addressing health equity from the perspectives of local populations requires that we examine the role of culture from positive, existential, and negative perspectives (C. O. Airhihenbuwa, 2007a) rather than focusing almost exclusively on the negative. Indeed, several public health leaders are demanding that the language of "disparity" be replaced with "equity" in order to offer a more promising perspective (such as social justice) and develop broad multidisciplinary partnerships to address growing disease burdens in local and international contexts (Braveman et al., 2011; Evans et al., 2001). Disparity has, in many cases, been confined to mean a focus solely on the comparative disadvantage of minority populations on a host of health indicators, whereas equity goes beyond quantitative differences to include how to reduce health gaps structurally and systematically to overcome the unfairness in health differences (Evans et al., 2001). Doing so requires developing a different mindset and engaging in capacity building for research with expectations for leadership, which is pivotal in research to address and promote health equity. Thus, each health researcher who is committed to addressing health inequity in the Global South must learn to claim his or her space.

\section{Claim Your Space}

Until the lions produce their own historians, the story of the hunt would glorify only the hunter. (African proverb)

Given the intractable burden of NCDs and IDs in the Global South, a recurring challenge is choosing a research and methodological direction and/or destination and figuring out how to get there. For the past 3 years, the lead author (COA) has been presenting lectures on leadership titled "Claim Your Space and Own Your Space" at different research capacity building forums that have involved all of the coauthors. This phrase quickly became a motto repeated by trainees and mentors alike as it articulated the need to produce "new historians" capable of responding to the question, "What should be the unique focus for capacity building for researchers in the Global South?" The strategies and approaches to conventional research capacity building tend to be based on training frameworks that view trainees as "the hands that receive," waiting for wisdom from the "hands that give." As Ahmadu Hampate Ba cautioned, "The hand that gives is always on top." Although it may be intentional, the institutional privilege of the hands that give tends to normalize trickle-down relationships, whereby the hands that receive silence their own voices as they learn to expect "wisdom" only from the hands that give. A new strategy requires giving/receiving to be perceived as 
sharing, whereby both hands occupy the same sharing and learning space, as opposed to the top-down position that typifies giver/receiver relationships.

As stated earlier, one of the major factors for success in scientific research that is often omitted in capacity building programs is leadership. Transformative scientific leadership can foster research with the goal of making a high impact on population health that is attentive to the needs and contexts of countries in the Global South. These goals have been the hallmark of national and international research capacity building projects briefly discussed earlier (CaRT and PRIDE) and led by the authors of this article. We have been working to develop a cadre of research scientists who will perform impactful population health research in their own local contexts. But one very important lesson we learned is that very few are trained to value leadership in their careers as scholars and scientists. Until we start training scholars in the global health in general, and Global South in particular, to become leaders in research, local capacity for high-quality research will not be strengthened. Countries in the Global South need a new breed of transformative leaders who are attuned to the research needs of their countries and committed to global health research priorities, particularly the double burden of NCDs and IDs. These global health priorities must be bold and highly innovative, with the potential to produce major impacts on population health and aimed at reducing multiple disease burdens in the region.

In addition, the Global South needs transformative leaders with a deep understanding of and commitment to research for social action. Transformative leaders possess an attitude of permanent openness as they engage in deeper, richer dialogues focused on learning from other people (Miller, Brown, \& Hopson, 2011; Shields, 2010). Their scholarship is marked by an intense faith in the inherent capabilities of all people to describe their realities and to transform them using solutions that translate local research into action (Carey, 1992). They critique inequitable power imbalances in collaborations with Global North researchers who often bring the resources and funding to the Global South with a promise to implement practices that are not ill-informed about the structural constraints of research in this region (Chu et al., 2014; Nchinda, 2002; Shields, 2010; Weiner, 2003).

Transformative leadership is inextricably related to capacity building for research in the Global South, and it holds promise for developing the next generation of research scientists grounded in the tradition advocated by Paulo Freire, "Learning to question." Instead of beginning with answers, the new historians should claim their spaces by questioning the question as they approach their research with the goal of developing sustainable solutions to NCDs in the Global South. The Global South also needs leaders who will embrace a research vision of an unquenchable desire for continuous critical engagement in global health research. Research capacity strengthening should involve nurturing transformative leaders from the Global South in ways that will reach the souls of others to raise human consciousness. This can create a favorable climate for researchers to own their spaces and make bigger policy impacts with transdisciplinary research approaches that hold better promise for reducing the multiple burdens of NCDs and IDs in the region. 


\section{Own Your Space}

No one should enter his house through another man's gate. (Chinua Achebe)

With transformative leadership comes the need for transformative knowledge and research visions in the Global South that are compelling and suitable for local priorities. Although NCDs and IDs coexist in Africa and the Global South, we simply cannot continue to enter the NCD world through the ID gate. In other words, we cannot address the challenges of the rising burden of NCDs in the Global South with a framework designed for IDs. The behavior, contexts, and institutional framework for addressing curable infections are very different from those that occasion diseases of lifestyle including consumption values and patterns. A new gate or perspective or framework is thus needed, even as we continue to acknowledge the prevailing importance of the ID gate from which important lessons should be learned. Moreover, any attempt to strengthen research capacity in the Global South must take into consideration the relationship between the local and the global spaces for risk situations and risk environments for NCDs relative to IDs. This means that researchers from the Global South must own their spaces of approaches to reducing NCDs that are anchored in their own space while cognizant of the influence of the global space. Local knowledge production echoes the value of multiple truths advanced by Chinua Achebe, who said, "When one thing stands, another thing stands beside it.... If there is one point of view, fine. There will be a second point of view." Normalizing the coexistence of otherwise different and divergent beliefs and ways of knowing remains the core essence of local reality in a global world for scholars in the Global South (C. O. Airhihenbuwa, 2007a). If training scientists is the key to research capacity strengthening, then we argue for a focus on transformative knowledge production based on transdisciplinary approaches that affirms unique "gates" for Global South scholars in ways that enable their research and scholarship to inform policies on health decisions that affect their local populations.

The search for different gates through which to give voice to the voiceless has led to the development of different strands of critical theories. Critical race theory is but one example of critical theory. In critical race theory, issues of structural and institutional racism are addressed so that the focus is not solely on individual behaviors but on the structures, systems, and policies that normalize marginalization of the "new historians" (Ford \& Airhihenbuwa, 2010a). Public health critical race praxis (Ford \& Airhihenbuwa, 2010b) is based on critical race theory. In public health critical race praxis, research capacity is designed to train early career researchers to employ a transdisciplinary approach to unpack the layers and levels of structural and institutional racism to be able to develop research agendas that are likely to have sustainable impact in promoting health equity among hitherto subpopulations with poor health outcomes (Ford \& Airhihenbuwa, 2010b).

The contributions of critical theory are far reaching. For example, Henry Giroux (1983) advanced the concept of critical pedagogy as a way to further extend notions of raising critical consciousness as introduced by Paulo Freire. Similar to other critical theories, critical pedagogy challenges us to recognize, engage, and critique (so as to transform) any existing practices and institutional structures that produce and sustain inequalities when building local research capacity (Giroux, 1983). Therefore, it is imperative that as we 
strengthen research capacity in these regions, we also need to provide scholars with the capacity to engage in critical analysis based on multidisciplinary knowledge production framework to benefit their local research agendas. Freire $(1998,2000)$ argued that engaging critical consciousness or conscientization (i.e., seeking to raise scholars' consciousness in order to unmask oppression and liberate the capacity to learn, imagine, act, and openly dialogue with the world) is also crucial to supporting local knowledge production through research.

Nonetheless, as we awaken new perspectives through research for health scholars in the Global South, we also need to deconstruct the Western model of science practiced by highincome-country researchers and currently transported to countries in the Global South (Chu et al., 2014). We need to change the continuing scientific colonialization of researchers in the Global South that involves high-income-country researchers flying into these countries to obtain data or samples and leaving with rich data that almost guarantees publications but no assurance of benefits of the research for the health of the population (Chu et al., 2014). In fact, many of these studies often fail because they have no commitment to sustainable solutions based on local knowledge and practices. We want to echo arguments advanced by several scholars (Mkandawire, 2005; Trostle \& Simon, 1992; Zeleza, 2002) who condemn scientific colonialism, which typically involves, among other activities, Global South only in data collection and no plan for training and infrastructure for data analysis and publications in these same populations. In many cases, the finished products (published articles) are shared in high-impact journals with no meaningful impact on the lives of the people in the countries that produced the raw data. This has led many to question what use is a publication in a peer-reviewed journal if the research does not have any direct local population benefit (Trostle \& Simon, 1992). Moreover, as C. O. Airhihenbuwa (2007a) argued in Healing Our Differences and as others have argued elsewhere, although the celebrated high-impact journals in biomedical and behavioral science have offered important contributions to knowledge production, these same journals have excluded new ways of knowledge production in other contexts, such as the health challenges in the Global South. Thus, a critique of knowledge production actively calls for a transformation of the terrain and parameters of scholarly inquiries in the Global South. We believe that a focus on transformative knowledge has the potential in practice to offer more inclusive, equitable scholarship that reconciles the identities of the populations that have the problems with the theories and models needed to frame solutions. Thus, to employ critical analysis of spaces that are claimed and owned is to reaffirm the continuing importance of those spaces in promoting health equity.

\section{Reaffirm Your Space}

We prefer self-government with danger to servitude in tranquility. ... We face neither east nor west; we face forward. (Kwame Nkrumah)

Not all researchers in the Global South carry out their missions with equal vigor, nor are they universally encouraged to develop their values as researchers (Nchinda, 2002). Thus, to strengthen research capacity while building transformative leaders that promote local knowledge production, reaffirming their spaces is critical for long-term sustainability. 
Scheirer and Dearing (2011) defined sustainability as the continued use of intervention components and activities for the continued achievement of desirable health outcomes within the population of interest. Historically, sustainability—whether in the form of measuring continued health benefits for individuals enrolled in interventions after initial funding ends, or continuing intervention activities within an organizational structure, or building the capacities of recipient communities to implement interventions themselves (Shediac-Rizkallah \& Bone, 1998) — has been a long-standing interest of academic researchers, governments, and the international donor community (Bossert, 1990). ShediacRizkallah and Bone (1998) suggested that termination of an intervention, particularly due to the expiration of funding, is counterproductive when the disease or health outcome remains or recurs. Many projects incur significant unexpected start-up costs in human, fiscal, and technical resources, only to see funds expire prior to the achievement of a predicted potential (Shediac-Rizkallah \& Bone, 1998).

Thus, we need to build research capacity in the Global South with the mindset that not only is knowledge inherently local but also that scientific representation of such local knowledge has special value for the long-term sustainability of interventions to address some of the pressing human health challenges in the region. No argument for research capacity strengthening in the region is going to have weight if it counters the need for sustainability. We believe that solving the dual burden of NCDs and IDs in the Global South will require a new kind of model that not only interrogates the theories and models that are used to guide the implementation of health research in the Global South but also continually demands and reaffirms the sustainability of the research implemented in the region. These factors share similarities with efforts to achieve technical and human research capacity strengthening in the Global South. Transformative leaders with a determination to define and prioritize what matters for their own context rather being tempted to follow the path of others, as illustrated in the quote above by Nkrumah, are more likely to seek innovative ways to sustain their research activities. For this to occur in the Global South, we must train the next generation of scientists to envision larger spaces for the next phases of their journeys and provide them with the skills necessary to sustain research programs in the region.

\section{Failure Is an Opportunity to Envision Your Next Space}

You may encounter defeat but you must not be defeated. (Maya Angelou)

The size of your dreams must always exceed your current capacity to achieve them. If your dreams do not scare you, they are not big enough. (President Ellen Johnson Sirleaf of Liberia)

An important component of leadership in research is learning to be comfortable with being uncomfortable (C. O. Airhihenbuwa, 2007a, 2007b). Indeed, reaffirmation of what we do is a launching platform for what will follow. What we do next must always be the result of a vision of health equity based on solutions developed from within. It is for this reason that failure must be embraced as an opportunity to envision the next space. To envision is to dream of possibilities that go beyond one's current position and location. To translate failure into a vision based on a dream, we must first address the challenges faced by scholars that must be overcome. 
Indeed, while a recurring theme in addressing health inequity globally is capacity building and strengthening, we often fail to answer the question, "For what purpose?" (Labonte \& Laverack, 2001; Trostle \& Simon, 1992). There are three major challenges for researchers in the Global South: increasing the number of skilled researchers who will build scholarly careers around performing research that will offer sustainable solutions to population health problems (Chu et al., 2014; Nchinda, 2002); anchoring research agendas on perspectives that are culturally relevant to local contexts so that sustainability can be ensured through ownership of solutions by the population; and helping donors, whether external or internal (including researchers' own governments), value the importance of investing in local solutions to local problems. At the intersection of these major challenges is failure, which we believe is an important component of success if scholars are to excel as leaders who are trained to question knowledge produced in their local contexts that is not based on local priorities or needs. Creating an opportunity for these research scientists to imagine their next space should also involve building a platform that proactively seeks to learn from research failures.

Failure is often defined as a deviation from expected and desired results that may include both avoidable and unavoidable outcomes (Cannon \& Edmondson, 2005). The reality is that transformative leaders of global health, in general, and the Global South, in particular, will experience failures as they face the numerous structural constraints for impactful and sustainable research in their region. Deviations from expected results due to flawed research designs or even publication/grant rejections may be viewed as failures, but even these failures present opportunities for learning. Learning from failures is the hallmark of innovative technology companies (Cannon \& Edmondson, 2005); and it is high time we value it in leadership for health research capacity building and strengthening. In fact, most researchers have an aversion to publicly acknowledging failures of any kind. Yet, for successful leaders and researchers, failure is an opportunity to envision new goals and, thus, must be considered inevitable in transformative leadership for global health researchers. Also, valuing failure must become a priority for funders whose research agendas may disrupt local medical and educational services or lead to detrimental effects on local population health and health care.

To strengthen research capacity, it is imperative that we create an awareness of the conditions that lead to failure, so that as these researchers fail, they understand that their failures to achieve expected outcomes are not always of their own making. Instead, such failures may be a function of myriad factors ranging from inadequate resources and misplaced research priorities to who traditionally dictates research agendas for the region (Chu et al., 2014; Nchinda, 2002; Trostle \& Simon, 1992). Reframing research failures as opportunities for learning is a critical first step in the journey to building and strengthening research capacity in the Global South.

Furthermore, researchers should learn not only from failures but also from successes in their own contexts. Very often, too much attention is focused on teaching research skills to solve problems, with little or no acknowledgement of positive aspects of their culture or contexts that may facilitate innovative solutions to population health. It has been previously argued that when you arrive in a community to address a health issue, particularly in the context of 
strengthening research capacity in the Global South to address NCDs and IDs, you should begin by recognizing something positive that the community does correctly (C. O.

Airhihenbuwa, 2007a, 2007b; Iwelunmor, Newsome, \& Airhihenbuwa, 2014). For example, local researchers can serve as role models and mentors to future research scientists. If you cannot identify something positive, then you should not remain in the community, otherwise you are likely to focus on research constraints, and may in fact become part of the problem (C. O. Airhihenbuwa, 2007a, 2007b; Iwelunmor et al., 2014). The act of beginning any research capacity strengthening by identifying success stories or positive aspects of research capacity in a community is central to the PEN-3 model, and should be at the core of any effort to train transformative global health researchers (C. O. Airhihenbuwa, 2007a; Iwelunmor et al., 2014).

\section{Conclusion}

Leadership is a core component of research capacity building. Leaders claim their spaces to understand and better appreciate levels and degrees of institutional and systematic processes that shape a successful research career. To this end, learning to claim and own one's space provides a critical foundation for a successful career in research and knowledge production. Beyond what is claimed and owned, each scholar must also reaffirm his or her own space and be prepared to envision a future direction. Perhaps most important, leaders must be prepared to deal with failure, yet never be defeated.

The next generation of global health scholars must include leaders who have the potential and determination to contribute to local, national, or international health priorities related to NCDs and IDs. For this to occur, resources must be invested to support a more effective goal of research capacity strengthening for health scholars in the Global South that focuses explicitly on leadership training, local knowledge production, sustainable research efforts, and learning from failures and successes. Unquestionably, there is a need for transformative leaders in global health from the Global South. However, transformation must move beyond the subtle implicit notion that there is no existing capacity in these settings (Mkandawire, 2005; Trostle \& Simon, 1992). We advocate for a mindset that provides opportunities for leaders to be led, just as those who are led are leaders (Weiner, 2003).

The challenge of limited resources has produced scholars who have become innovative in developing ideas and solutions to problems in their local communities, and leadership and capacity building must provide space for these innovations to be unleashed. It is in the midst of difficulties that many great ideas and great leaders have emerged. This is so clearly captured in the African expression, "Smooth seas do not make skillful sailors." We believe that research capacity strengthening needs to be oriented not just as a strategy but situated within the culture and values of the trained scientists in a specific region.

We call for a strong leadership that values transdisciplinary methods to build research capacity in the Global South. As researchers, we must commit ourselves to research capacity strengthening activities that not only strengthen but also inspire health scholars to make a difference in their local contexts. There is urgency in promoting transformative leadership as we build and strengthen research capacity that is sustainable and balances two fundamental 
values: excellence in research and relevance, and leadership in setting local research agendas. Indeed, promoting leadership must be a goal when preparing researchers who are committed to addressing local and national health needs and priorities in the Global South.

\section{Acknowledgments}

\section{Funding}

The authors disclosed receipt of the following financial support for the research, authorship, and/or publication of this article: Drs Ogedegbe and Jean-Louis were supported in part by the following grants from the National Institutes of Health (R25HL105444) and the NIH Fogarty International Center D43TW009140. Dr Ogedegbe was also supported by grants K24HL111315 and U01HL114198.

\section{References}

Airhihenbuwa C, Okoror T, Shefer T, Brown D, Iwelunmor J, Smith E, Dlakulu R. Stigma, culture, and HIV and AIDS in the Western Cape, South Africa: An application of the PEN-3 cultural model for community-based research. Journal of Black Psychology. 2009; 35:407-432. [PubMed: 22505784]

Airhihenbuwa, CO. Healing our differences: The crisis of global health and the politics of identity. Lanham, MD: Rowman \& Littlefield; 2007a.

Airhihenbuwa CO. On being comfortable with being uncomfortable: centering an Africanist vision in our gateway to global health. Health Education \& Behavior. 2007b; 34:31-42. [PubMed: 17200092]

Airhihenbuwa CO, Shisana O, Zungu N, BeLue R, Makofani DM, Shefer T, Simbayi L. Research capacity building: A US-South African partnership. Global Health Promotion. 2011; 18(2):27-35.

Bossert TJ. Can they get along without us? Sustainability of donor-supported health projects in Central America and Africa. Social Science \& Medicine. 1990; 30:1015-1023. [PubMed: 2336568]

Braveman PA, Kumanyika S, Fielding J, LaVeist T, Borrell LN, Manderscheid R, Troutman A. Health disparities and health equity: The issue is justice. American Journal of Public Health. 2011; 101(Suppl. 1):S149-S155. [PubMed: 21551385]

Cannon MD, Edmondson AC. Failing to learn and learning to fail (intelligently): How great organizations put failure to work to innovate and improve. Long Range Planning. 2005; 38:299_ 319.

Carey MR. Transformational leadership and the fundamental option for self-transcendence. Leadership Quarterly. 1992; 3:217-236.

Chu KM, Jayaraman S, Kyamanywa P, Ntakiyiruta G. Building research capacity in Africa: Equity and global health collaborations. PLoS Medicine. 2014; 11(3):e1001612. [PubMed: 24618823]

Drain PK, Primack A, Hunt DD, Fawzi WW, Holmes KK, Gardner P. Global health in medical education: A call for more training and opportunities. Academic Medicine. 2007; 82:226-230. [PubMed: 17327707]

Evans, T., Whitehead, M., Diderichsen, F., Bhuiya, A., Wirth, M., Whitehead, M. Challenging inequities in health: From ethics to action. Oxford, England: Oxford University Press; 2001.

Ford CL, Airhihenbuwa CO. Critical race theory, race equity, and public health: Toward antiracism praxis. American Journal of Public Health. 2010a; 100(Suppl. 1):S30-S35. [PubMed: 20147679]

Ford CL, Airhihenbuwa CO. The public health critical race methodology: Praxis for antiracism research. Social Science \& Medicine. 2010b; 71:1390-1398. [PubMed: 20822840]

Freire, P. Pedagogy of freedom: Ethics, democracy, and civic courage. Lanham, MD: Rowman \& Littlefield; 1998.

Freire, P. Pedagogy of the oppressed. London, England: Bloomsbury; 2000.

Geneau R, Hallen G. Toward a systemic research agenda for addressing the joint epidemics of HIV/ AIDS and noncommunicable diseases. AIDS. 2012; 26(Suppl):S7-S10. [PubMed: 22781179]

Giroux $H$. Theories of reproduction and resistance in the new sociology of education: A critical analysis. Harvard Educational Review. 1983; 53:257-293. 
Guilbert J. The World Health Report 2006: Working together for health. Education for Health (Abingdon, England). 2006; 19:385-387.

Hunter DJ, Reddy KS. Noncommunicable diseases. New England Journal of Medicine. 2013; 369:1336-1343. [PubMed: 24088093]

Iwelunmor J, Newsome V, Airhihenbuwa CO. Framing the impact of culture on health: A systematic review of the PEN-3 cultural model and its application in public health research and interventions. Ethnicity \& Health. 2014; 19(1):20-46. [PubMed: 24266638]

Jean-Louis G, Ayapa I, Rapoport D, Zizi F, Airhienbuwa C, Okuyemi K, Ogedegbe G. Mentoring junior URM scientists to engage in sleep health disparities research: Experience of the NYU PRIDE institute. Sleep Medicine. 2015; Advance online publication. doi: 10.1016/j.sleep. 2015.09.010

Labonte R, Laverack G. Capacity building in health promotion, Part 1: For whom? And for what purpose? Critical Public Health. 2001; 11:111-127.

Langer A, Díaz-Olavarrieta C, Berdichevsky K, Villar J. Why is research from developing countries underrepresented in international health literature, and what can be done about it? Bulletin of the World Health Organization. 2004; 82:802-803. [PubMed: 15643806]

Lansang MA, Dennis R. Building capacity in health research in the developing world. Bulletin of the World Health Organization. 2004; 82:764-770. [PubMed: 15643798]

Mayosi BM. The 10 "best buys" to combat heart disease, diabetes and stroke in Africa. Heart. 2013; 99:973-974. [PubMed: 23680892]

Miller PM, Brown T, Hopson R. Centering love, hope, and trust in the community transformative urban leadership informed by Paulo Freire. Urban Education. 2011; 46:1078-1099.

Mkandawire, T. African intellectuals and nationalism. London, England: Zed Books; 2005.

Naghavi M, Forouzanfar MH. Burden of noncommunicable diseases in sub-Saharan Africa in 1990 and 2010: Global Burden of Diseases, Injuries, and Risk Factors Study 2010. Lancet. 2013; 381:S95.doi: 10.1016/S0140-6736(13)61349-5

Narayan KV, Miotti PG, Anand NP, Kline LM, Harmston C, Gulakowski R III, Vermund SH. HIV and noncommunicable disease comorbidities in the era of antiretroviral therapy: A vital agenda for research in low-and middle-income country settings. Journal of Acquired Immune Deficiency Syndromes. 2014; 67(Suppl. 1):S2-S7. [PubMed: 25117958]

Nchinda TC. Research capacity strengthening in the South. Social Science \& Medicine. 2002; 54:1699-1711. [PubMed: 12113452]

Ogedegbe G, Gyamfi J, Plange-Rhule J, Surkis A, Rosenthal DM, Airhihenbuwa C, Cooper R. Task shifting interventions for cardiovascular risk reduction in low-income and middle-income countries: A systematic review of randomised controlled trials. BMJ Open. 2014; 4(10):e005983.

Ogedegbe G, Plange-Rhule J, Gyamfi J, Chaplin W, Ntim M, Apusiga K, Cooper R. A clusterrandomized trial of task shifting and blood pressure control in Ghana: Study protocol. Implementation Science. 2014; 9(1):73. [PubMed: 24923300]

Raeburn J, Akerman M, Chuengsatiansup K, Mejia F, Oladepo O. Community capacity building and health promotion in a globalized world. Health Promotion International. 2006; 21(Suppl. 1):84-90. [PubMed: 17307961]

Scheirer MA, Dearing JW. An agenda for research on the sustainability of public health programs. American Journal of Public Health. 2011; 101(11):2059-2067. [PubMed: 21940916]

Shediac-Rizkallah MC, Bone LR. Planning for the sustainability of community-based health programs: Conceptual frameworks and future directions for research, practice and policy. Health Education Research. 1998; 13(1):87-108. [PubMed: 10178339]

Shields CM. Transformative leadership: Working for equity in diverse contexts. Educational Administration Quarterly. 2010; 46:558-589.

Trostle J, Simon J. Building applied health research capacity in less-developed countries: Problems encountered by the ADDR project. Social Science \& Medicine. 1992; 35:1379-1387. [PubMed: 1462177]

Uthman OA, Wiysonge CS, Ota MO, Nicol M, Hussey GD, Ndumbe PM, Mayosi BM. Increasing the value of health research in the WHO African region beyond 2015-Reflecting on the past, 
celebrating the present and building the future: A bibliometric analysis. BMJ Open. 2015; 5(3):e006340.

Weiner EJ. Secretary Paulo Freire and the democratization of power: Toward a theory of transformative leadership. Educational Philosophy and Theory. 2003; 35(1):89-106.

Zeleza PT. The politics of historical and social science research in Africa. Journal of Southern African Studies. 2002; 28(1):9-23.

Ziraba AK, Fotso JC, Ochako R. Overweight and obesity in urban Africa: A problem of the rich or the poor? BMC Public Health. 2009; 9(1):465. [PubMed: 20003478] 